\title{
Correction to: Anterior cruciate ligament reconstruction with concomitant meniscal surgery: a systematic review and meta- analysis of outcomes
}

\author{
Mohamed Sarraj ${ }^{1} \cdot$ Ryan P. Coughlin ${ }^{2} \cdot$ Max Solow $^{3} \cdot$ Seper Ekhtiari ${ }^{2} \cdot$ Nicole Simunovic $^{2} \cdot$ Aaron J. Krych $^{4}$. \\ Peter MacDonald ${ }^{5}$. Olufemi R. Ayeni ${ }^{2,6}$ (D)
}

Published online: 13 March 2019

(c) European Society of Sports Traumatology, Knee Surgery, Arthroscopy (ESSKA) 2019

\section{Correction to: \\ Knee Surgery, Sports Traumatology, Arthroscopy \\ https://doi.org/10.1007/s00167-019-05389-3}

Unfortunately, the middle name of Olufemi R. Ayeni was accidentally omitted in the original publication and the author name is corrected here. The original article has been corrected.

Publisher's Note Springer Nature remains neutral with regard to jurisdictional claims in published maps and institutional affiliations.

The original article can be found online at https://doi.org/10.1007/ s00167-019-05389-3.

Olufemi R. Ayeni

ayenif@mcmaster.ca

Mohamed Sarraj

mohamed.sarraj@medportal.ca

Ryan P. Coughlin

ryan.coughlin@medportal.ca

Max Solow

maxsolow@live.ca

Seper Ekhtiari seper.ekhtiari@medportal.ca

Nicole Simunovic

simunovic@mcmaster.ca

Aaron J. Krych

krych.aaron@mayo.edu

Peter MacDonald

PMACDONALD@panamclinic.com
DeGroote School of Medicine, McMaster University, Hamilton, Canada

2 Division of Orthopaedic Surgery, Department of Surgery, McMaster University, Hamilton, Canada

3 St. George's University, University Centre Grenada, West Indies, Grenada

4 Department of Orthopedic Surgery and the Sports Medicine Center, Mayo Clinic and Mayo Foundation, Rochester, USA

5 Department of Surgery, University of Manitoba, Winnipeg, Canada

6 Department of Health Research Methods, Evidence, and Impact, McMaster University Medical Centre, Hamilton, Canada 